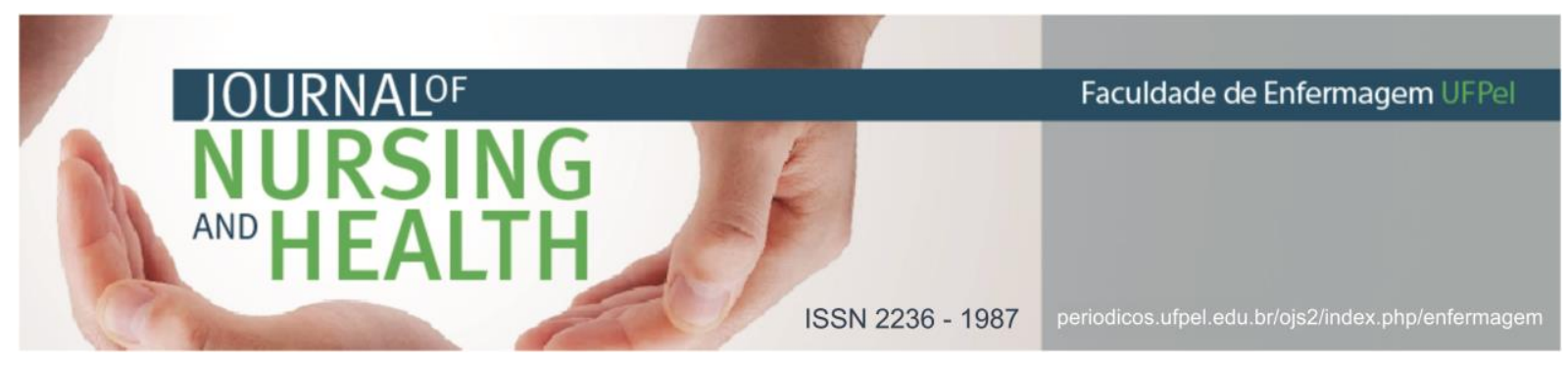

ARTIGO ORIGINAL

\title{
Vivência de enfermeiras(os) acerca dos incidentes relacionados à administração de medicamentos em terapia intensiva
}

\author{
Nurses' experience regarding incidents of administration of medicines in intensive \\ care
}

\section{Vivencia de enfermeras(os) acerca de incidentes relacionados a la administración de medicamentos en terapia intensiva}

Costa, Milena Pereira ${ }^{1}$; Picanço, Carina Marinho²; Batalha, Edenise Maria Santos da Silva ${ }^{3}$

Como citar esse artigo: Costa MP, Picanço CM, Batalha EMSS. Vivência de enfermeiras(os) acerca dos incidentes relacionados à administração de medicamentos em terapia intensiva. J. nurs. health. 2018;8(2):e188207

\section{RESUMO}

Objetivo: avaliar a vivência das(os) enfermeiras(os) em relação aos incidentes de segurança do paciente na administração de medicamentos em Unidades de Terapia Intensiva. Método: estudo quantitativo, realizado com enfermeiras(os) de um hospital de Salvador, Bahia. Os dados foram coletados por meio de questionário estruturado e analisados por meio de estatística descritiva. Resultados: todos os participantes referiram ter presenciado um incidente na administração de medicamento. 0 tipo mais frequente foi dosagem errada. Os fatores contribuintes mais citados foram falta de atenção e sobrecarga de trabalho. As condutas mais tomadas após o incidente apontam para a observação do quadro clínico e comunicação à coordenação e os fatores dificultadores para a notificação foram medo da punição e de julgamento. Conclusão: é preciso unir esforços tanto individual quanto no âmbito da gestão para que as ações sejam direcionadas no intuito de reduzir os incidentes de segurança de paciente.

Descritores: Segurança do paciente; Erros de medicação; Enfermeiras e enfermeiros; Unidades de terapia intensiva.

\begin{abstract}
Objective: to evaluate the experience of nurses in relation to the patient safety incidents in the administration of drugs in Intensive Care Units. Method: a quantitative study performed with nurses from a hospital in Salvador, Bahia. Data were collected through a structured questionnaire and analyzed using descriptive statistics. Results: all participants reported having seen an incident in medication administration. The most frequent type was wrong dosage. The most cited contributing factors were lack of attention and work overload. The behaviours most taken after the incident point to the observation of the clinical picture and communication to the coordination and the factors that made it difficult to notify were fear of punishment and of judgment. Conclusion: both individual and management efforts must be combined to ensure that actions are taken to reduce patient safety incidents.

Descriptors: Patient safety; Medication errors; Nurses; Intensive care units.

1 Enfermeira. Residente em Terapia Intensiva. Universidade do Estado da Bahia (UNEB). E-mail: myllena_costa@hotmail.com http://orcid.org/0000-0002-2645-1331

2 Enfermeira. Mestre em Enfermagem. Hospital Geral Roberto Santos, Salvador/BA. E-mail: carinampicanco@gmail.com http://orcid.org/0000-0003-4758-4333

3 Enfermeira. Mestre em Enfermagem. Universidade do Estado da Bahia (UNEB). E-mail: edenisemaria@gmail.com http://orcid.org/0000-0003-0014-9529
\end{abstract}




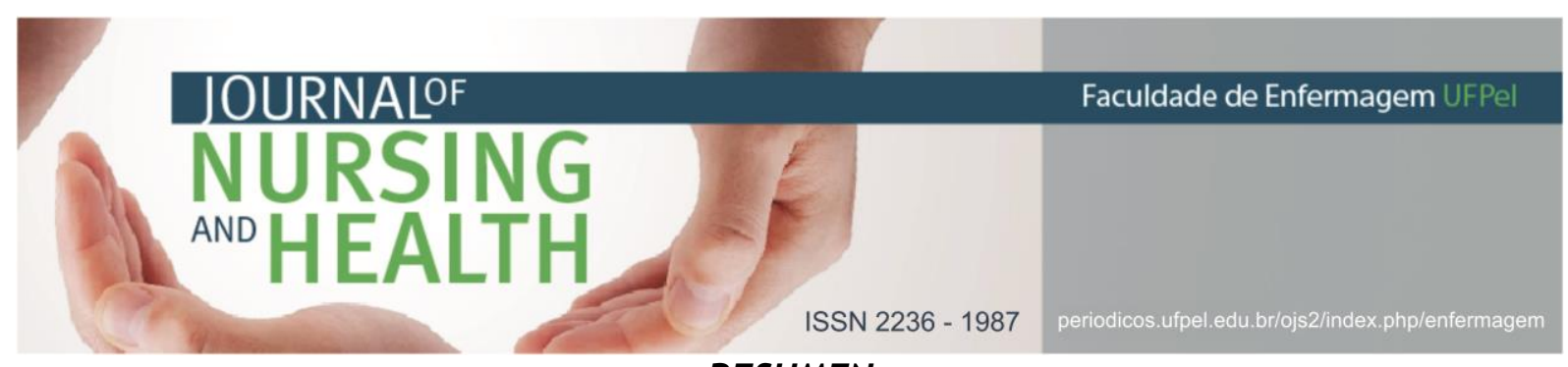

RESUMEN

Objetivo: evaluar la vivencia de las enfermeras en relación a los incidentes de seguridad del paciente en la administración de medicamentos en Unidades de Terapia Intensiva. Método: estudio cuantitativo, realizado con enfermeras de un hospital de Salvador, Bahia. Los datos fueron recolectados por medio de un cuestionario estructurado y analizados por medio de estadística descriptiva. Resultados: todos los participantes indicaron haber presenciado un incidente en la administración de medicamentos. El tipo más frecuente fue dosificación incorrecta. Los factores contribuyentes más citados fueron falta de atención y sobrecarga de trabajo. Las conductas más tomadas después del incidente apuntan la observación del cuadro clínico y comunicación a la coordinación y los factores dificultadores para la notificación fueron miedo del castigo y juicio. Conclusión: es necesario unir esfuerzos tanto individual como en el ámbito de la gestión para que las acciones se dirijan a fin de reducir los incidentes de seguridad del paciente.

Descriptores: Seguridad del paciente; Errores de medicación; Enfermeros; Unidades de cuidados intensivos.

\section{INTRODUÇÃO}

A administração de medicamentos é uma atividade inerente à prática assistencial de enfermagem em Unidades de Terapia Intensiva (UTI). Os fármacos contribuem de forma significativa para a melhoria do estado de saúde das pessoas trazendo-lhes benefícios sociais e econômicos, mas seu uso não é isento de risco.

Os erros de medicação estão conceituados como "qualquer evento evitável, que pode causar ou levar ao uso inadequado do medicamento ou a danos ao paciente enquanto 0 medicamento está sob o controle de profissionais de saúde, do paciente ou do consumidor". 2:26

De acordo com o Ministério da Saúde (MS), os erros de medicação podem ser classificados em: erro de prescrição, definido como um erro de redação ou decisão; erro de dispensação, relacionados com as atividades da farmácia hospitalar e erros de administração, qualquer desvio no preparo e administração do medicamento. ${ }^{3}$
O erro no preparo e na administração do fármaco pode ocorrer e não ser identificado e nem valorizado pela equipe de enfermagem, bem como as graves repercussões que este pode gerar para os pacientes. ${ }^{4}$

Nesse contexto, a Agência de Vigilância Sanitária define incidente como "um evento ou circunstância que poderia ter resultado, ou resultou, em dano desnecessário à saúde". 5:36 Os incidentes classificam-se como: near miss (incidente que não atingiu o paciente); incidente sem dano (evento que atingiu o paciente, mas não causou dano discernível) e incidente com dano ou Evento Adverso (EA) (incidente que resulta em dano ao paciente). ${ }^{6}$

Nas UTI esses incidentes e suas consequências para $o$ indivíduo e equipe de enfermagem merecem um enfoque especial. A vulnerabilidade dos pacientes, a complexa terapia medicamentosa, a utilização de uma grande quantidade de medicamentos e realização de procedimentos, associados à carga de trabalho da enfermagem e o número insuficiente 


\section{JOURNALOF \\ NURSING \\ AND HEALTH}

ISSN 2236 - 1987

de profissionais justifica a frequência dos $\operatorname{erros}^{7}$ e a necessidade de uma análise mais focalizada.

Assim a enfermagem precisa atuar na busca por identificar as possíveis falhas no sistema de saúde, e solucionar os problemas advindos, oferecendo assistência segura e efetiva, livre de qualquer dano durante a prestação do cuidado. ${ }^{8}$

Ao detectar erros no seu ambiente de trabalho, o profissional deve exercer o seu papel de educador, compartilhar e interagir com a equipe, possibilitando interpretar as ocorrências do sistema, para que estratégias sejam traçadas buscando o alcance de melhorias. ${ }^{9}$ Além disso, é importante a notificação dos incidentes, pois é um método que possibilita conhecimento dos erros e elaboração de relatórios, permitindo aprendizado e indução de mudança de comportamentos. ${ }^{10}$

Neste cenário estudar os
incidentes de segurança na administração de medicamentos, tem como relevância a possibilidade de contribuir para o conhecimento relacionado à sua ocorrência e sua importância, tanto no âmbito das organizações hospitalares, quanto para profissionais de saúde, em especial os enfermeiros, bem como uma reflexão sobre o impacto financeiro e assistencial para a instituição e o dano causado aos pacientes, influenciando no seu tratamento.

Devido às poucas produções que existem sobre a vivência dos profissionais e seus relatos sobre os incidentes, na tentativa de propor melhorias na qualidade da assistência para todos os envolvidos nesse processo, justifica-se a realização do estudo, que teve como objetivo geral: avaliar a vivência das (os) enfermeiras (os) em relação aos incidentes de segurança do paciente na administração de medicamentos em Unidades de Terapia Intensiva. E, como objetivos específicos: identificar os fatores relacionados à ocorrência destes incidentes; descrever as condutas adotadas perante os incidentes; e identificar os desafios para a notificação dos mesmos.

\section{MATERIAIS E MÉTODOS}

Estudo de caráter quantitativo, descritivo e exploratório. Constituíram participantes do estudo 55 enfermeiros atuantes nas UTI de um Hospital Geral, público, da cidade de Salvador Bahia. 0 cálculo amostral foi realizado através do software Epilnfo $7^{\mathrm{TM}} \mathrm{com}$ base no número total de trabalhadores e teve como resultado 62 profissionais, porém não foi obtido retorno de todos os questionários entregues. Os critérios de inclusão foram: ser enfermeira (o) e trabalhar nas UTI adulto do referido hospital. Os critérios de exclusão: estarem de licença ou férias no período de coleta de dados.

A pesquisa foi realizada apenas com enfermeiros, dado que no campo de estudo a administração de medicamentos nas UTI é realizada de forma prioritária por essa categoria e não pelos técnicos de enfermagem. A coleta de dados foi realizada nas UTI de adultos do referido hospital com capacidade total de 48 leitos. Os dados foram coletados no período de julho a setembro de 2017, através de questionário estruturado composto de 
questões objetivas sobre dados sociodemográficos (Parte I: 9 questões) e questões relacionadas a situações vivenciadas ou presenciadas relacionadas a incidentes de segurança na administração de medicamentos, condutas tomadas pelos profissionais e desafios para a notificação na percepção das(os) enfermeiras(os) (Parte II-5 questões). Continha também informações sobre o preenchimento do questionário, bem como a definição e classificação de incidentes de segurança do paciente.

Os questionários foram entregues aos participantes nas unidades referidas, posteriormente ao Termo de Consentimento Livre e Esclarecido (TCLE) e coletados após concordância em participar, assinatura do TCLE e preenchimento do instrumento.

A análise dos dados se deu através de estatística descritiva. Foi utilizado o programa Microsoft $®$ Excel 2010 para tabulação e criação de gráficos e tabelas.

A pesquisa foi aprovada pelo Comitê de Ética em Pesquisa da Universidade do Estado da Bahia (UNEB), protocolo de $\mathrm{n}^{\circ}$ 2.102.394. 0 estudo foi realizado em conformidade com os princípios de autonomia, beneficência, não maleficência, justiça e equidade versadas na Resolução $n^{\circ} 466$, de 12 de dezembro de 2012, do Conselho Nacional de Saúde, que trata das normas e diretrizes regulamentadoras de pesquisas envolvendo seres humanos.

\section{RESULTADOS}

A Tabela 1 refere-se ao perfil sócio-demográfico e profissional dos participantes.

Quando questionados sobre terem vivenciado ou presenciado um incidente relacionado à administração de medicamentos na sua vida profissional, todos (100\%) os enfermeiros referiram sim. Quanto à frequência que isso foi visto ou vivenciado, mais da metade disseram que ocorreu mais de três vezes.

Os tipos de incidentes relatados pelos enfermeiros, em maior frequência, foram: dose incorreta $(17,4 \%)$, seguido de diluição incorreta $(16,2 \%)$. Alguns participantes $(n=14$; $25,5 \%$ ) referiram que houve dano ao paciente, conforme a Tabela 2 .

A falta de atenção $(23,1 \%)$ seguido da sobrecarga de trabalho $(18,3 \%)$ foram os fatores contribuintes mais citados. Conforme a análise dos dados, verificou-se que os enfermeiros indicaram um total de 112 condutas tomadas diante da ocorrência do incidente. Quanto às condutas realizadas após o incidente, a intervenção medicamentosa foi prevalente $(61,0 \%)$, como descrito na Tabela 3.

Em relação aos fatores dificultadores para a notificação dos incidentes, pelas(os) enfermeiras(os), o medo da punição obteve maior frequência, citado por 38 profissionais $(33,3 \%)$, em seguida o medo do julgamento da equipe (30,7\%), conforme a Figura 1. 
Tabela 1: Perfil sócio-demográfico e profissional dos participantes

\begin{tabular}{|c|c|c|}
\hline IDADE & $\mathrm{n}$ & $\%$ \\
\hline $23-29$ anos & 18 & 32,7 \\
\hline $30-40$ anos & 28 & 50,9 \\
\hline Acima de 40 anos & 9 & 16,4 \\
\hline \multicolumn{3}{|l|}{ SEXO } \\
\hline Feminino & 48 & 87,3 \\
\hline Masculino & 7 & 12,7 \\
\hline \multicolumn{3}{|l|}{ ESTADO CIVIL } \\
\hline Solteiro (a) & 29 & 52,7 \\
\hline Casado (a) & 20 & 36,4 \\
\hline Divorciado (a) & 3 & 5,5 \\
\hline Viúvo (a) & 2 & 3,6 \\
\hline Não respondeu & 1 & 1,8 \\
\hline \multicolumn{3}{|l|}{ VÍNCULO PROFISSIONAL } \\
\hline Servidor efetivo & 17 & 30,9 \\
\hline Vínculo temporário & 9 & 16,4 \\
\hline Celetistas e cooperativistas & 19 & 34,6 \\
\hline Residentes & 8 & 14,5 \\
\hline Não Respondeu & 2 & 3,6 \\
\hline \multicolumn{3}{|l|}{ MAIS DE UM EMPREGO } \\
\hline Sim & 29 & 52,7 \\
\hline Não & 26 & 47,3 \\
\hline \multicolumn{3}{|l|}{ TEMPO DE FORMADO } \\
\hline $\begin{array}{l}1 \text { ano } \\
2 \text { a } 4 \text { anos } \\
4 \text { a } 6 \text { anos } \\
>6 \text { anos }\end{array}$ & $\begin{array}{c}4 \\
20 \\
9 \\
22 \\
\end{array}$ & $\begin{array}{c}7,2 \\
36,4 \\
16,4 \\
40,0 \\
\end{array}$ \\
\hline \multicolumn{3}{|l|}{ TEMPO DE SERVIÇO EM UTI } \\
\hline $\begin{array}{l}<1 \text { ANO } \\
1 \text { ano } \\
2 \text { a } 4 \text { anos } \\
4 \text { a } 6 \text { anos } \\
>6 \text { anos } \\
\text { Não respondeu }\end{array}$ & $\begin{array}{c}7 \\
8 \\
17 \\
7 \\
15 \\
1 \\
\end{array}$ & $\begin{array}{c}12,7 \\
14,5 \\
31,0 \\
12,7 \\
27,3 \\
1,8 \\
\end{array}$ \\
\hline \multicolumn{3}{|c|}{ PÓS-GRADUAÇÃO EM TERAPIA INTENSIVA } \\
\hline $\begin{array}{l}\text { Sim } \\
\text { Não } \\
\text { Não respondeu }\end{array}$ & $\begin{array}{c}38 \\
15 \\
2\end{array}$ & $\begin{array}{c}69,1 \\
27,3 \\
3,6\end{array}$ \\
\hline
\end{tabular}


Tabela 2: Distribuição das respostas dadas pelas (os) enfermeiras nos questionários aplicados Salvador/2018

\begin{tabular}{lcc}
\hline VIVENCIOU OU PRESENCIOU INCIDENTE & $\mathbf{n}$ & $\%$ \\
\hline Sim & 55 & 100,0 \\
\hline QUAL TIPO? & & \\
\hline Dose incorreta & 29 & 17,4 \\
Diluição incorreta & 27 & 16,2 \\
Medicação errada & 25 & 15,0 \\
Omissão de dose & 25 & 15,0 \\
Paciente errado & 20 & 12,0 \\
Horário incorreto & 19 & 11,4 \\
Via incorreta & 16 & 9,5 \\
Medicamento alérgeno & 6 & 3,5 \\
\hline QUANTAS VEZES? & & \\
\hline Acima de três & 31 & 56,4 \\
Uma & 9 & 16.4 \\
Duas & 7 & 12,7 \\
Três & 6 & 10,9 \\
Não respondeu & 2 & 3,6 \\
\hline HOUVE DANO? & & \\
\hline Não & 40 & 14,3 \\
Sim & 14 & 72,7 \\
Não respondeu & 1 & 25,5 \\
\hline QUAL O TIPO DE DANO? & & 1,8 \\
\hline Alteração padrão ventilatório e/ou cardíaco & 8 & \\
Sonolência & 4 & 19,0 \\
Óbito & 3 & \\
Reação Alérgica & 3 & \\
Agitação & 3 & \\
\hline & & \\
\hline
\end{tabular}




\section{NURSING

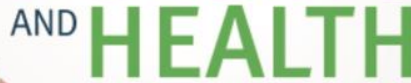

ISSN 2236 - 1987

Tabela 3: Distribuição das respostas dadas pelas(os) enfermeiras(os) nos questionários aplicados Salvador/2018

\begin{tabular}{lcc}
\hline FATORES CONTRIBUINTES & N & $\%$ \\
\hline Falta de atenção & 39 & 23,1 \\
Sobrecarga de trabalho & 31 & 18,3 \\
Falta de conhecimento & 26 & 15,4 \\
Pressa & 23 & 13,6 \\
Cansaço & 19 & 11,2 \\
Erro de prescrição & 12 & 7,2 \\
Erro de dispensação & 7 & 4,1 \\
Polifarmácia & 7 & 4,1 \\
Paciente inconsciente & 3 & 1,8 \\
Material inadequado administração & 2 & 1,2 \\
\hline CONDUTAS ADOTADAS & & \\
\hline Observação do quadro & 35 & $31,2 \%$ \\
Comunicou à coordenação & 25 & $22,3 \%$ \\
Comunicou ao médico & 18 & $16,1 \%$ \\
Comunicou à equipe & 16 & $14,3 \%$ \\
Notificou & 14 & $12,5 \%$ \\
Não comunicou a ninguém & 4 & $3,6 \%$ \\
\hline INTERVENÇÕES REALIZADAS & & \\
\hline Medicamentosa & 20 & 61,0 \\
Laboratoriais & 8 & 24,0 \\
Reanimação & 3 & 9,0 \\
Intubação & 2 & 6,0 \\
\hline
\end{tabular}

Figura 1: Percentual dos fatores dificultadores para notificação dos incidentes de segurança do paciente relacionados à administração de medicamentos.

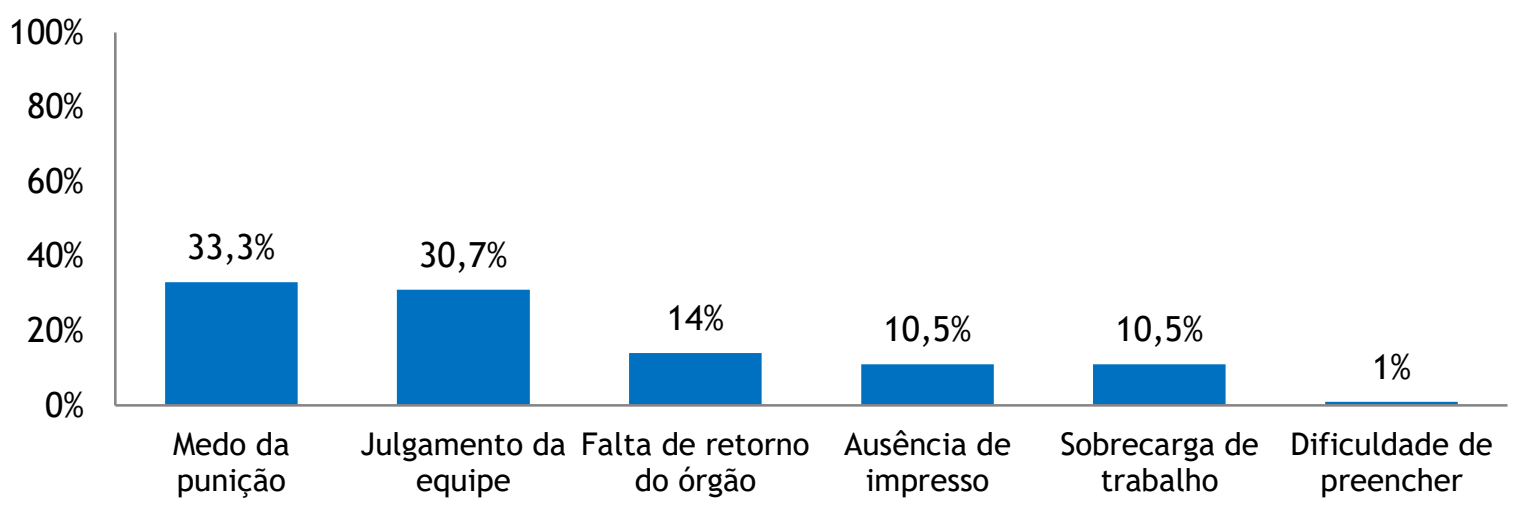




\section{DISCUSSÃO}

Dentre os profissionais que atuam na cadeia medicamentosa, os enfermeiros, público alvo do presente estudo, os quais estão envolvidos nas etapas finais do sistema de medicação, por unanimidade, referiram já terem vivenciado ou presenciado um incidente com medicamento em sua carreira profissional, a maior parte deles por mais de três vezes, o que revela ser, possivelmente, um fato frequente na assistência à saúde de pacientes gravemente enfermos. Assim, houve uma falha em alguma das etapas que compõem a cadeia medicamentosa acarretando em incidente, o qual poderia ser evitado.

Um estudo transversal que teve como um dos objetivos identificar a prevalência de erros de medicação em UTI de um hospital privado, constatou que dos 94 profissionais participantes, $18(19,1 \%)$ mencionaram ter cometido erros de medicação durante as quatro semanas anteriores à coleta de dados, sendo 06 enfermeiros e 12 técnicos. ${ }^{11}$ Resultado esse inferior ao obtido nesta pesquisa, que pode ser justificado pelo fato de objetivarmos também os casos que foram presenciados pelo profissional, não apenas àqueles que foram vivenciados por eles, como também, por não termos instituído tempo para a vivência do incidente.

Quanto aos tipos de incidentes relatados pelas(os) enfermeiras(os), os mais frequentes foram àqueles caracterizados por dosagem errada e diluição incorreta. 0 impacto disso pode ter uma dimensão maior, visto que se trata de pacientes com gravidade clínica, utilizando medicamentos complexos e que doses diferentes da recomendada, assim como a diluição incorreta que eleva ou diminui a concentração da droga, podem causar sérias complicações, como exemplo a insuficiência renal.

Nesse caso é imprescindível a presença de um farmacêutico clínico nas UTI, a instituição e utilização de protocolos de diluição de medicamentos, assegurando seu uso correto.

A dosagem do medicamento, corroborando com este estudo, também foi o principal erro encontrado em $27(67,5 \%)$ dos 40 estudos analisados em uma pesquisa do tipo revisão integrativa, que objetivou identificar as evidências e as implicações dos erros na administração de medicamentos na segurança do paciente. $^{12}$

Numa análise mais detalhada em um estudo exploratório-descritivo de um Hospital público, os tipos de erros durante a administração de medicamentos cometidos por profissionais de enfermagem foram em ordem de frequência: falhas relacionadas às normas de biossegurança 25 (55,6\%); horário de administração errado $21 \quad(46,7 \%)$; omissão dose 18 (40,0\%); velocidade de infusão errada 17 (37,8\%); técnica de administração errada 16 (35,6\%); não monitoração do paciente após medicação $14 \quad(31,1 \%)$; via de administração errada 12 (26,7\%); medicamento administrado em paciente errado $11 \quad(24,4 \%)$; medicamento administrado errado 09 (20,0\%); administração de medicamento não prescrito $08(17,8 \%)$, entre outros. ${ }^{9}$ 


\section{JOURNALOF \\ NURSING \\ ANO HEALTH}

ISSN 2236 - 1987

de atenção e sobrecarga de trabalho. A desatenção $(57,7 \%)$ foi a principal causa de erro elencada por enfermeiros notificadores de UTI e Unidades de internação, de um estudo descritivo-exploratório documental, realizado em um Hospital de ensino. ${ }^{15}$ Em trabalho qualitativo realizado por Santana e outros, que teve como um dos objetivos identificar os fatores que propiciam os erros de enfermagem em uma Unidade de Terapia Intensiva na percepção da equipe de enfermagem destaca o cansaço, a sobrecarga de trabalho, a má remuneração e quantidade insuficiente de profissionais como fatores citados pelos entrevistados. ${ }^{16}$

Os profissionais envolvidos na administração de medicamentos precisam estar atentos em todo o processo e serem capazes de identificar situações que propiciem a ocorrência de incidentes, pois, sabendo-se que um incidente pode causar danos aos pacientes e prolongar o tempo de internação, como revelou um estudo prospectivo onde os pacientes com EA tiveram maior tempo médio de internação na UTI, quando comparados àqueles que não sofreram $(p \leq 0,000),{ }^{17}$ esse cuidado é imprescindível.

No tocante à sobrecarga de trabalho, um estudo tipo coorte, que visou identificar a influência da sobrecarga de trabalho da Enfermagem na ocorrência de incidentes sem lesão e eventos adversos em pacientes de UTI em dois hospitais Brasileiros, demonstrou que a gravidade clínica (pontuação Apache II) na admissão a UTI $(0,35 ; \quad \mathrm{p}<0,0001)$, a carga de trabalho da Enfermagem (Nursing 
Activities Score - NAS) 0,191 e $\mathrm{p}<0,0001$ e o peso das comorbidades (pontuação escala de Charlson), 0,128 e $\mathrm{p}<0,0001$, apresentaram uma correlação significativa com a ocorrência de EA, revelando a necessidade de um dimensionamento adequado do quadro de pessoal desta categoria profissional. ${ }^{18}$

Sabe-se que essa é uma questão bastante discutida entre os profissionais de saúde que frequentemente acumulam mais de um vínculo, a exemplo dos profissionais desta pesquisa onde mais da metade possui mais de um emprego, em consequência da baixa remuneração oferecida pelos empregadores, além da ausência de piso salarial para a categoria. Portanto, acumula também estresse, sono, cansaço, falta de concentração que podem impactar na assistência prestada aos pacientes, bem como nas outras funções gerenciais, de capacitação e orientação exercidas pelos enfermeiros.

Vale ressaltar que a falta de conhecimento e a inexperiência em terapia intensiva, citada por alguns enfermeiros do estudo, contribuem para o aumento da incidência desses eventos. Daí destaca-se o papel da educação permanente, uma estratégia que vai promover o desenvolvimento e aprimoramento dos profissionais de saúde, associadamente a um número adequado de profissionais visando à redução de carga horária, na garantia de um cuidado seguro e qualificado, livre de danos tanto ao paciente quanto aos trabalhadores, sendo uma condição que deve ser oferecida pelas instituições hospitalares.
Nessa perspectiva, evidenciam-se as sugestões fornecidas pelos profissionais para prevenir erros de medicação, como o treinamento e a capacitação dos funcionários apontados por $150(92,6 \%)$ profissionais e a diminuição da sobrecarga de trabalho indicada por $112 \quad(69,1 \%)$ trabalhadores em um estudo quantitativo, descritivo exploratório que buscou identificar erros cometidos pelos profissionais de enfermagem em relação à administração medicamentosa, reiterando a necessidade de educação permanente e capacitação, já que a educação e supervisão contínua representam fatores importantes para uma assistência integral e de qualidade ${ }^{19}$.

Quanto às condutas tomadas pelos profissionais diante dos incidentes evidenciou-se que a maioria relatou observação do quadro clínico. Diante disso, chama a atenção o fato dos trabalhadores não comunicarem a ocorrência de incidentes, nem notificarem, como foi observado nos resultados. Diferente deste cenário, um estudo que teve por objetivo identificar a ocorrência de erros na administração de medicamentos relacionada à prática da equipe de enfermagem em um hospital de pequeno porte, obteve como condutas mais citadas pelos participantes após a ocorrência de erro na administração de medicamentos: comunicam 0 enfermeiro 15 (15,8\%); não comunicam o erro $13(13,7 \%)$; agem como se nada tivesse acontecido $13 \quad(17,3 \%)$; intensificam os cuidados 12 (12,6\%); comunicam o médico responsável 10 (10,5\%); conversam com o colega sobre o ocorrido 9 (9,5\%); comunicam o médico plantonista $8(8,4 \%){ }^{20}$ 


\section{ISSN $2236-1987$}

A comunicação ao médico só foi citada por $8,4 \%$ dos participantes do estudo acima apesar de ser devida, visto que poderão ser necessárias medidas como a prescrição de outros fármacos para evitar maiores consequências após o incidente. Podese constatar a partir dos resultados que a notificação do evento também não foi realizada, pois não houve nenhum relato nas condutas tomadas pelos participantes.

O medo da punição, o julgamento da equipe e a falta de retorno do órgão responsável pelos aspectos notificados foram os mais citados pelos participantes dessa pesquisa como fatores dificultadores para a notificação dos incidentes. Corroborado em outro estudo ${ }^{21}$ tal resultado, quando afirmam que a subnotificação pode ocorrer por diversos fatores incluindo o medo, a culpa e também o tipo de sistema de notificação.

Apesar de não ter sido o fator dificultador que obteve maior frequência, o medo de punição $(21,2 \%)$ também foi apontado em outra pesquisa ${ }^{22}$ como motivo para ausência de notificação de eventos adversos e, enfermeiros relataram haver medidas punitivas aplicadas pelas instituições quando há ocorrência de EA.

As instituições de saúde, atualmente, são recomendadas a criarem um comitê em segurança do paciente, desenvolvendo a cultura de segurança com foco na não punição e implementação de recomendações voltadas para as melhores práticas, identificação, notificação e análise dos incidentes, assim reduzindo o número desses $^{23}$, bem como a subnotificação.
Vale ressaltar que a cultura de segurança do paciente deve circundar os trabalhadores que têm contato direto com o paciente, bem como a gestão hospitalar, mobilizando-os a exercer tal cultura com efetividade, buscando atitudes e comportamentos pautados no compromisso com a segurança, mantendo uma comunicação com o nível estratégico, político e operacional da instituição. ${ }^{24}$

\section{CONCLUSÃO}

A ocorrência de incidentes de segurança na administração de medicamentos em UTI representa um sério problema com relação à qualidade da assistência e promoção de um ambiente seguro ao paciente. Este estudo evidenciou que todos os participantes já vivenciaram ou presenciaram um incidente desse tipo, o que comprova ser fator frequente e preocupante na assistência ao paciente crítico.

0 tipo que obteve maior frequência foi o relacionado à dosagem errada. Os fatores contribuintes mais citados foram falta de atenção e sobrecarga de trabalho. Sobre as condutas tomadas após o incidente a maioria dos participantes apontam para a observação do quadro clínico e comunicação à coordenação de enfermagem. Em relação aos fatores dificultadores para a notificação, os mais referidos foram medo da punição e julgamento da equipe.

A partir desses resultados nota-se uma necessidade de educação permanente, investimento em pessoal, bem como melhorias salariais, com o objetivo do aprimoramento profissional, da redução de sobrecarga 


\section{JOURNALOF \\ NURSING \\ ANO HEALTH}

ISSN 2236 - 1987

com medicamentos no contexto hospitalar. Cad saude pública (online) [Internet]. 2008 set[acesso em 2018 set 25];24(9):1965-75. Disponível em: http://www.scielo.br/pdf/csp/v24n9 /02.pdf

2 Cousins DD. Developing a uniform reporting system for preventable adverse drug events. Clinical theraputics [Internet]. 1998[cited 2018 $\begin{array}{lll}\text { Setp 25]; 20(suppl C):C45-59. } & \text { 20 }\end{array}$ Available from: https: / / linkinghub.elsevier.com/retri eve/pii/S0149-2918(98)80008-8

3 Ministério da Saúde (BR). Conselho regional de farmácia do estado de São Paulo. Farmácia hospitalar. Brasília; 2012.

4 Barros AP. Práticas no preparo e administração de medicamentos em unidade de terapia intensiva neonatal e pediátrica: uma pesquisa-ação [tese]. Recife (PE): Universidade Federal de Pernambuco; 2015.

5 Ministério da Saúde (BR). Agência Nacional de Vigilância Sanitária. Implantação do núcleo de segurança do paciente em serviços de saúde - série segurança do paciente e qualidade em serviços de saúde. Brasília; 2014.

6 World health organization (WHO). Conceptual framework for the international classification for patient safety;2009.

7 Forte ECN, Machado FL, Pires DEP. A relação da enfermagem com os erros de medicação: uma revisão integrativa. Cogitare enferm [Internet]. 2016[acesso em 2018 set 25];21(n.esp):01-10. Disponível em: https://revistas.ufpr.br/cogitare/arti cle/view/43324/pdf

1 Rissato MAR, Romano-lieber NS, Lieber RR. Terminologia de incidentes 
8 Dias JD, Mekaro KS, Tibes CMS, ZemMascarenhas SH. Compreensão de enfermeiros sobre segurança do paciente e erros de medicação. REME rev min enferm [Internet]. 2014[acesso em 2018 set 25]; 18(4):866-73. Disponível em: http://www.reme.org.br/artigo/detal hes $/ 969$

9 Galiza DDF, Moura OF, Barros VL, Luz GOA. Preparo e administração de medicamentos: erros cometidos pela equipe de enfermagem. Revista brasileira de famácia hospitalar e serviços de saúde [Internet]. 2014[acesso em 2018 set 24];5(2):4550. Disponível em: http://www.sbrafh.org.br/rbfhss/pub lic/artigos/2014050205000528BR.pdf

10 Lorenzini E, Santi JAR, Bao ACP. Segurança do paciente: análise dos incidentes notificados em um hospital do sul do Brasil. Rev gaúch enferm [Internet]. 2014[acesso em 2018 set 24];35(2):121-7. Disponível em: http://www.seer.ufrgs.br/RevistaGau chadeEnfermagem/article/view/4437 $0 / 29940$

11 Pelliciotti JSS, Kimura M. Erros de medicação e qualidade de vida relacionada à saúde de profissionais de enfermagem em unidades de terapia intensiva. Rev latinoam enferm (online) [Internet]. 2010[acesso em 2018 jan 27];18(6). Disponível em: http://www.scielo.br/pdf/rlae/v18n6 /pt_04.pdf

12 Gomes ATL, Assis YMS, Silva MF, Costa IKF, Feijão AR, Santos VEP. Erros na administração de medicamentos: evidencias e implicações na segurança do paciente. Cogitare enferm [Internet]. 2016[acesso em 2018 set
24];21(3):01-11. Disponível em: http://docs.bvsalud.org/biblioref/201 6/11/2729/44472-186904-1-pb.pdf

13 Borges MC, Faria JIL, Jabur MRL, Oliveira KA, Zborowski IP, Beccaria LM. Erros de medicação e grau de dano ao paciente em hospital escola. Cogitare enferm [Internet]. 2016[acesso em 2018 set 24];21(4):01-09. Disponível em:

https://revistas.ufpr.br/cogitare/arti cle/view/45397/pdf

14 Cassiani SHB, Gimenes FRE, Monzani AAS. O uso da tecnologia para a segurança do paciente. Rev eletrônica enferm [Internet]. 2009[acesso em 2018 fev 20];11(2):413-7. Disponível em:

https://www.fen.ufg.br/revista/v11/ n2/pdf/v11n2a24.pdf

15 Vilela RPB, Jericó MC. Erro de medicação: gestão do indicador para uma prática mais segura. Rev enferm UFPE online [Internet]. 2016[acesso em 2018 jan 25];10(1):119-27. Disponível em: https://periodicos.ufpe.br/revistas/r evistaenfermagem/article/download/ 10929/12220

16 Santana JCB, Miranda FL, Gonçalves LM, Foureaux PV, Almeida SJ, Duarte CC. latrogenias na assistência em uma unidade de terapia intensiva: percepção da equipe de enfermagem. Enferm rev [Internet]. 2015; maio/ago[acesso em 2018 fev 05];18(2). Disponível em: http://periodicos.pucminas.br/index. php/enfermagemrevista/article/view/ 11690/10334

17 Roque KE, Melo ECP. Adaptação dos critérios de avaliação de eventos adversos a medicamentos para uso em 


\section{ISSN 2236 - 1987}

um hospital público no estado do Rio de Janeiro. Rev bras epidemiol [Internet]. 2010[acesso em 2018 set 25];13(4):607-19. Disponível em: http://www.scielo.br/pdf/rbepid/v13 n4/06.pdf

18 Novaretti MCZ, Santos EV, Quitério LM, Daud-Gallotti RM. Sobrecarga de trabalho da enfermagem e incidentes e eventos adversos em pacientes internados em uti. Rev bras enferm [Internet]. 2014 set/out[acesso em 2018 set 25];67(5):692-99. Disponível em:

http://www.scielo.br/pdf/reben/v67 n5/0034-7167-reben-67-05-0692.pdf

19 Silva MVRS, Carvalho FSSF, Branco TB, Guimarães JTF, Lima NDP, Alves AKC. Administração de medicamentos: erros cometidos por profissionais de enfermagem e condutas adotadas. Rev enferm UFSM [Internet]. 2018 set/mar[acesso em 2018 set 15];8(1):102-15. Disponível em: https://periodicos.ufsm.br/reufsm/ar ticle/view/25686/pdf

20 Vestena CFL, Girardon-Perlini NMO, Rosa BVC, Stamm B, Beuter M, Rosa N. Erros na administração de medicamentos: estudo com uma equipe de enfermagem. Rev enferm UFPI [Internet]. 2014 out/dez[acesso em 2018 fev 21];3(4):42-9. Disponível em:

http://www.ojs.ufpi.br/index.php/re ufpi/article/view/2293

21 Capucho HC, Arnas ER, Cassiani SHBD. Segurança do paciente: comparação entre notificações voluntárias manuscritas e informatizadas sobre incidentes em saúde. Rev gaúch enferm [Internet]. 2013[acesso em 2018 set 25];
34(1):164-72. Disponível em: http://www.scielo.br/pdf/rgenf/v34n 1/21.pdf

22 Ferezin TPM, Ramos D, Caldana G, Gabriel CS, Bernardes A. Análise da notificação de eventos adversos em hospitais acreditados. Cogitare enferm [Internet]. 2017[acesso em 2018 set 25]; 22(2). Disponível em: https: / / revistas.ufpr.br/cogitare/arti cle/view/49644/pdf_en

23 Teixeira TC, Cassiani SH. Análise de causa raiz de acidentes por quedas e erros de medicação em hospital. Acta paul enferm (online) [Internet]. 2014[acesso em 2018 jan 12] 27(2):100-7. Disponível em: http://www.scielo.br/pdf/ape/v27n2 /0103-2100-ape-27-02-0100.pdf

24 Batalha EMSS, Melleiro MM. Cultura de segurança do paciente em um hospital de ensino: diferenças de percepção existentes nos diferentes cenários dessa instituição. Texto \& contexto enferm [Internte]. 2015[acesso em 2018 set 25];24(2):432-41. Disponível em: http://www.scielo.br/pdf/tce/v24n2/ pt_0104-0707-tce-24-02-00432.pdf

Data de submissão: 16/04/2018

Data de aceite: 07/09/2018

Data de publicação: 30/09/2018 